\title{
Cellulite d'origine dentaire engageant le pronostic vital : à propos d'un cas
}

\author{
Cellulitis of dental origin with serious complications. Report of a case
}

CHARLES DUBERNARD ${ }^{1,3}$, SANDRA BELLANGER ${ }^{1,3}$, GUILLAUME CHAMBON², HERVE LÉON ${ }^{1}$, JACQUES-HENRI TORRES ${ }^{1}$, JEAN LOZZA ${ }^{1}$

\section{RÉSUMÉ}

L'extension au médiastin des cellulites diffuses d'origine dentaire constitue une complication rare mais extrêmement grave. Le cas rapporté est celui d'un patient de 25 ans ayant développé une cellulite cervico-faciale à partir d'une troisième molaire mandibulaire. II a présenté un choc septique avec syndrome de détresse respiratoire aiguë. L'examen tomodensitométrique a montré un emphysème cervico-scapulo-médiastinal. Paradoxalement, le sujet était resté apyrétique, et le bilan biologique a retrouvé un taux de leucocytes normal à un stade avancé de l'infection, ce qui fait évoquer la notion d'un terrain prédisposant.

Le patient a bénéficié d'une prise en charge lourde faisant intervenir de nombreuses techniques médicales et chirurgicales, pour suppléer d'une part les défaillances multi-viscérales et d'autre part, traiter l'infection par antibiothérapie et drainage (cervicotomie et thoracotomie).

Après 3 mois de coma, le patient conservait principalement des séquelles fonctionnelles bucco-faciales et des cicatrices cutanées dues au drainage cervical et thoracique. (Med Buccale Chir Buccale 2009 ; 15: 119-125).

mots clés: cellulite, médiastinite, réanimation

médecine buccale chirurgie buccale VOL. $15, \mathrm{~N}^{\circ} 3$ 2009 page 119

\section{SUMMARY}

The mediastinal extension of diffuse cellulitis of dental origin is a rare but extremely serious complication. The case of a 25 years old male patient who developed a cervico-facial cellulitis starting from a mandibular third molar is reported. He presented a septic shock, with respiratory distress. The CTscan showed a cervico-scapulo-mediastinal emphysema. The patient paradoxically remained apyretic and his leukocyte rate remained normal at an advanced stage of the infection. These findings evoke an infection-prone background.

The patient underwent a heavy management including numerous medical and surgical techniques, to make up for the multivisceral failure on one hand, and on the other hand to treat the infection through antibiotic therapy and drainage (cervicotomy, thoracotomy).

After 3 months' coma, the patient will keep oro-facial after-effects and cutaneous scares related to the drainage (Med Buccale Chir Buccale 2009 ; 15: 119-125).

key words: cellulitis, mediastinitis, intensive care

\footnotetext{
1. Service d'Odontologie CHU Nîmes

2. Service d'ORL et de Chirurgie maxillofaciale CHU Nîmes

3. Service d'Odontologie CHU Montpellier
}

Demande de tirés à part:

Charles Dubernard 8 rue Fournarié 34000 Montpellier charlesdubernard@hotmail.com 
Les cellulites cervico-faciales constituent la majorité des infections graves d'origine dentaire. Leur extension au médiastin représente une complication rare, pouvant mettre en jeu la vie du patient. Cet article rapporte un cas dont l'évolution a été particulièrement sévère.

\section{OBSERVATION}

Un homme de 25 ans, d'origine comorienne, sans antécédent médical ni chirurgical particulier, a été admis aux urgences du $\mathrm{CHU}$ de Nimes dans un état d'angoisse et de malaise général. Bien qu'apyrétique, il présentait une dyspnée de décubitus et une dysphagie complète. Trois jours auparavant, il avait consulté un chirurgien-dentiste pour une infection siégeant sur la 38 (Fig. 1) ; ce dernier lui avait prescrit une antibiothérapie (amoxicilline, $3 \mathrm{~g} / \mathrm{jour}$ ), de l'ibuprofène, du paracétamol et un bain de bouche antiseptique à la chlorhexidine.

médecine

buccale

chirurgie

buccale

VOL. $15, \mathrm{~N}^{\circ} 3$

2009

page 120
Lors de l'examen clinique, le médecin urgentiste a retrouvé un œdème du plancher buccal associé à un trismus serré rendant l'examen difficile, un empâtement cervical bilatéral et un emphysème souscutané étendu de la région cervicale à la région thoracique antérieure, avec crépitation neigeuse à la palpation. Toutefois, l'évaluation dermatologique, bien que rendue difficile par la pigmentation cutanée du sujet, n'a pas retrouvé d'aspect inflammatoire de la peau.

Le bilan biologique effectué était en faveur d'un sepsis sévère, avec une augmentation de la $\mathrm{C}$ réactive protéine (CRP > $300 \mathrm{mg} / \mathrm{L}$ ), une insuffisance rénale modérée, une acidose métabolique et des créatines phosphokinases élevées évoquant une nécrose musculaire. Mais paradoxalement, il n'y avait pas d'hyperleucocytose et le patient restait apyrétique. Le bilan scannographique réalisé en urgence a mis en évidence l'étendue de l'emphysème (Fig. 2).

Le traitement initial a consisté en une antibiothérapie intra-veineuse : amoxicilline $5 \mathrm{~g} / \mathrm{j}$ et métronidazole 2g/j. La dyspnée s'est aggravée et un état de choc est apparu. Devant la sévérité du tableau clinique, le patient a été transféré au service de réanimation, où une sédation a été mise en place avec ventilation assistée ('intubation a été difficile en raison du refoulement des tissus mous). Un arrêt cardiaque a nécessité un massage cardiaque externe et une injection intra-veineuse d'adrénaline, à la suite de laquelle l'activité cardiaque a rapidement récupéré. Le patient a ensuite été admis en urgence au

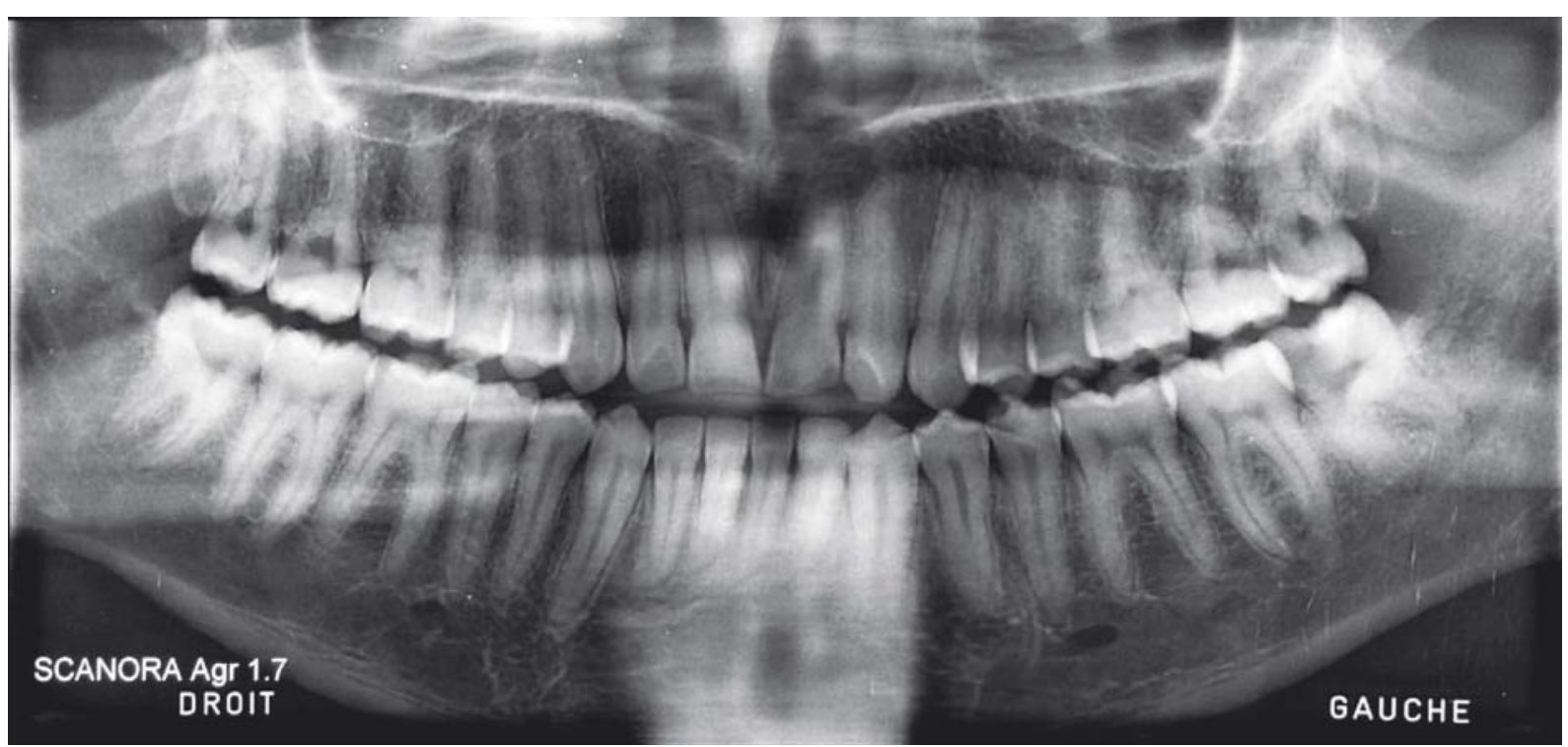

Figure 1 : Orthopantomogramme, examen complémentaire de choix pour rechercher une étiologie dentaire. Ici, la 38 présente une lésion carieuse mésiale étendue.

Panoramic radiography, effective further test to search a dental carie. In this case, the 38 presents a wide mesial decay. 


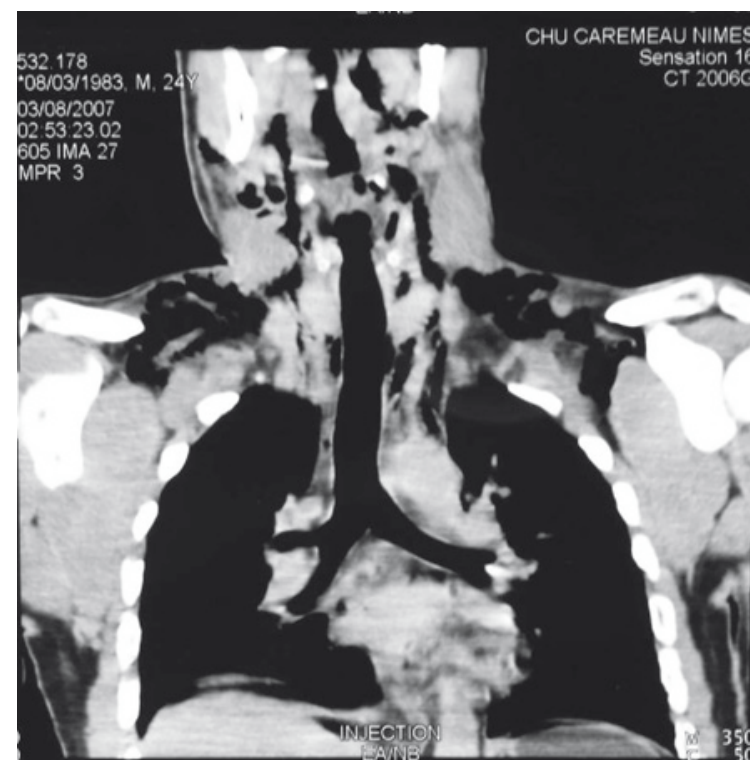

Figure 2 : Coupe frontale cervico-thoracique objectivant l'emphysème cervico-scapulo-médiastinal. Frontal cervico-thoracic section which shows the cervico-scapulo-mediastinal emphysema.

bloc opératoire ORL pour débridement et drainage de l'infection. Une cervicotomie large avec abord de l'ensemble des loges cervicales a mis en évidence un aspect de cellulite gangréneuse avec une nécrose tissulaire extensive. Cette nécrose atteignait le plancher buccal, les gouttières vasculaires jugulo-carotidiennes, les creux sus-claviculaires, les espaces para- et rétro-pharyngés et le médiastin antérieur (Fig. 3). Les prélèvements bactériens réalisés en per-opératoire ont révélé une association d'entérobacters aérogènes, et de streptocoques

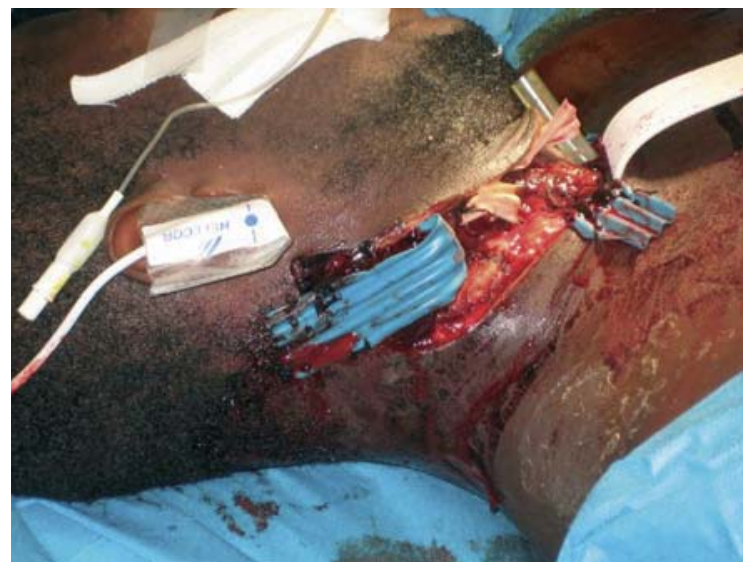

Figure 3 : Cervicotomie de drainage. Draining cervical incision.

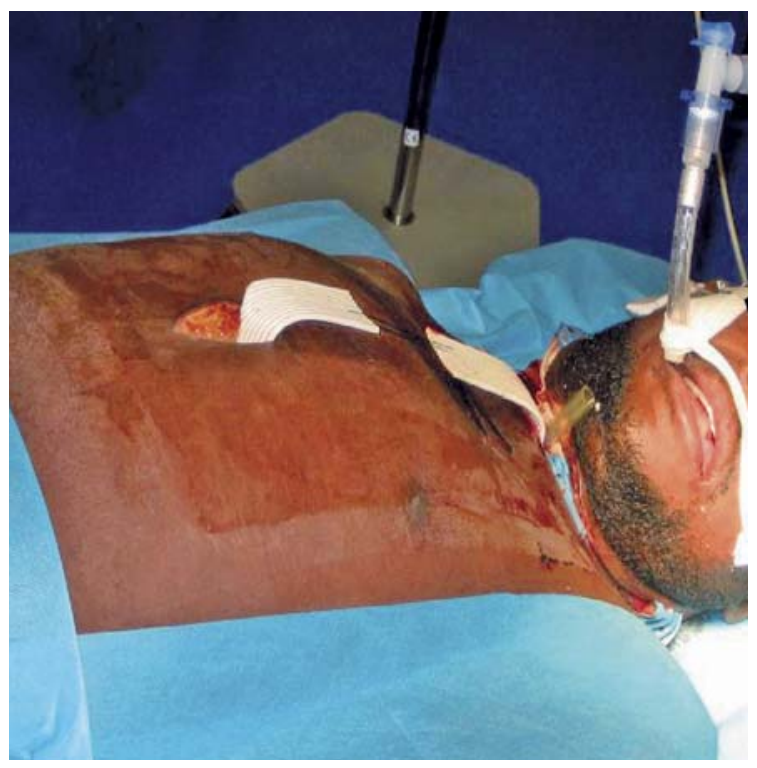

Figure 4 : Drainage du médiastin antérieur avec lame rétro-sternale.

Draining mediastinal anterior incision with retro-sternal drain.

aérobies et anaérobies. Dans le même temps, les chirurgiens vasculaires ont procédé à un abord du médiastin avec mise en place d'une lame rétro-sternale (Fig. 4). Les jours suivants, plusieurs reprises chirurgicales ont été nécessaires pour le drainage d'une pleurésie purulente.

Rapidement, la situation a évolué sur le mode d'une défaillance multiviscérale, avec choc septique, syndrome de détresse respiratoire aiguë, insuffisance rénale aiguë nécessitant des séances de dialyse, et hypothyroïdie transitoire attribuée aux lésions nécrotiques locales.

Des lésions dermatologiques nécrotiques sont aussi apparues aux chevilles (Fig. 5).

Après trois semaines d'hospitalisation, le patient a bénéficié d'une trachéotomie et d'une jéjunostomie d'alimentation. Durant trois mois, les médecins ORL ont pratiqué de nombreux drainages et lavages au sérum physiologique additionné de povidone iodée tous les deux à trois jours. L'état général s'est lentement amélioré.

Enfin, à la sortie du service de réanimation, lorsque l'état de santé était stabilisé, l'avulsion de la 38 causale a été pratiquée.

A sa sortie, le patient présentait des séquelles : atteinte des nerfs spinal (XI) et grand hypoglosse médecine buccale chirurgie buccale VOL. $15, \mathrm{~N}^{\circ} 3$ 2009 page 121 
(XII) gauches avec limitation de la mobilité cervicale et linguale (Fig. 6) ainsi qu'une insuffisance rénale modérée.

Une motivation à l'hygiène dentaire a été menée, ainsi que des contrôles bucco-dentaires réguliers.

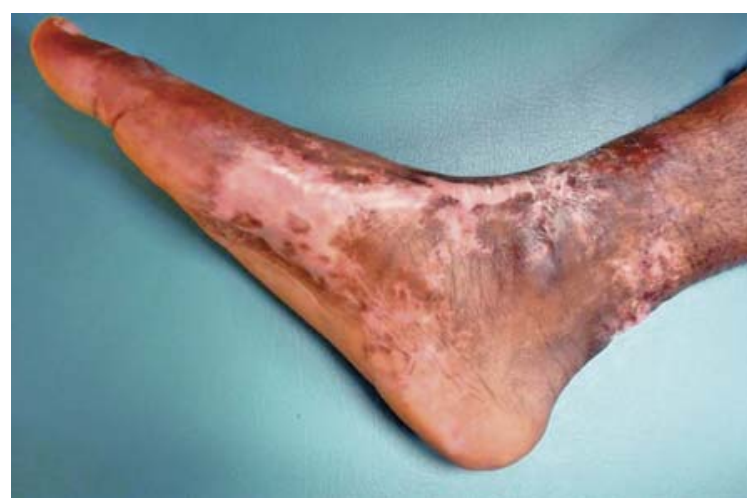

Figure 5 : Aspect du pied au $5^{\mathrm{e}}$ mois avec des lésions cutanées nécrotiques en cours de cicatrisation. Ces lésions ont résulté soit d'une localisation septique secondaire, soit d'une coagulation intra-vasculaire disséminée. L'étiologie médicamenteuse a été écartée du fait du caractère localisé des lésions.

Foot's photography at 5th month showing the healing of cutaneous necrotic lesions. Those lesions is either the result of a secondary septic localisation or the result of a disseminated intravascular

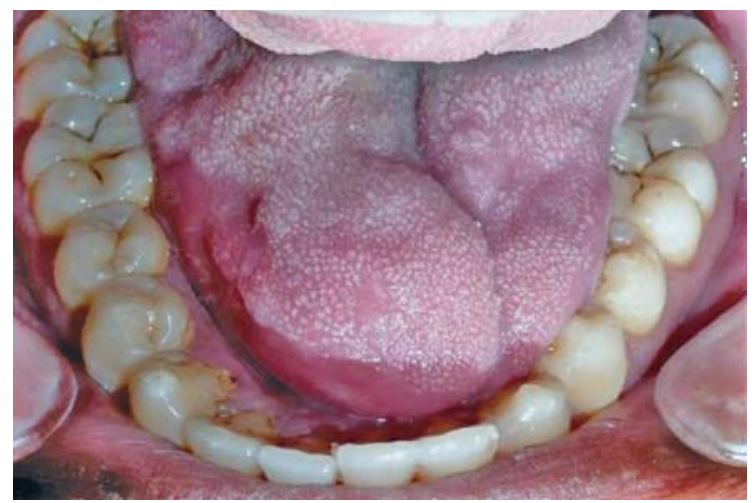

Figure 6 : Déviation de la langue à gauche due à la lésion du nerf grand hypoglosse gauche.

Lingual deviation to the left, linked to the lesion of the left hypoglossal nerve.

\section{COMMENTAIRES}

La cellulite d'origine dentaire est une infection polymicrobienne des tissus cellulo-adipeux remplissant les loges qui jouxtent la mandibule et le maxillaire. Cette infection a une tendance extensive. En effet, les loges communiquent d'abord entre elles, notamment par l'intermédiaire de l'espace para-amygdalien, puis avec les grands espaces anatomiques de décollement qui s'étendent de la base du crâne jusqu'au médiastin. La médiastinite constitue alors la complication la plus redoutée. Elle entraîne souvent une septicémie. A ce stade tardif, l'issue peut être fatale malgré une prise en charge adaptée. Dans le cas rapporté, le décès semble avoir été évité de justesse, en particulier lors de la déficience du système circulatoire.

Les formes nécrosantes gravissimes représentent moins de $10 \%$ des cellulites cervico-faciales d'après Romain et coll. [1]. Mais selon Rakotoarison et coll. [2], leur fréquence serait en augmentation. La plupart des auteurs notent une prédominance pour le sexe masculin : $71 \%$ des cas pour Rakotoarison et coll. [2], $66 \%$ des cas pour Romain et coll. [1]. Les formes graves se retrouvent en particulier chez les sujets jeunes, comme dans le cas rapporté ici.

Peron et Mangez ${ }^{[3]}$ rappellent que l'affaiblissement des défenses de l'hôte peut jouer un rôle déterminant dans la survenue d'une infection bucco-dentaire. II peut s'agir de facteurs physiologiques (grossesse, carence nutritionnelle, etc.), de facteurs environnementaux (traumatisme physique ou psychique, etc.) ou d'un déficit immunitaire congénital ou acquis (SIDA, traitement immunosuppresseur, diabète, insuffisance hépatocellulaire d'origine virale ou alcoolique). Mais à l'inverse, Scheffer et coll. [4] insistent sur le fait qu'il peut s'agir assez souvent de sujets sains sans antécédent pathologique. Dans le cas rapporté, deux éléments sont en faveur d'un dysfonctionnement immunitaire : l'apyrexie et le taux de leucocytes resté normal à un stade déjà avancé de l'infection. Un déficit immunitaire congénital apparaît pourtant peu probable étant donné que ces pathologies se révèlent généralement à un âge bien plus précoce. Par ailleurs, le bilan sérologique n'a mis en évidence aucune sérologie d'infection virale pouvant induire un déficit immunitaire, en particulier une infection par le VIH. Bien que des portes d'entrées pharyngées, sinusiennes et cutanées aient été rapportées dans la littérature ${ }^{[2]}$, les voies de pénétration infectieuse sont principalement dentaires et parodontales. 
Peron et Mangez ${ }^{[3]}$ évoquent la nécrose pulpaire (d'origine carieuse ou traumatique), l'infection parodontale (principalement les péricoronarites des troisièmes molaires mandibulaires) mais également les lésions endo-parodontales. Les dents le plus souvent mises en cause dans les cellulites aiguës diffuses sont les dents postérieures mandibulaires. A partir de l'espace desmodontal, l'infection traverse l'os, décolle le périoste, puis le rompt et colonise les parties molles péri-osseuses qui sont constituées par un tissu cellulo-adipeux remplissant les espaces délimités par les zones d'insertion musculo-aponévrotiques des tables osseuses médiale et latérale. Parallèlement, il peut exister une propagation par voie veineuse et lymphatique dépassant les barrières anatomiques locales. A la mandibule, les deux dernières molaires ont leurs apex situés contre la table osseuse médiale et audessous de la ligne d'insertion du muscle mylohyoïdien. Aussi, les infections qui en sont issues peuvent-elles ensemencer directement la région cervicale. En l'occurrence, le point de départ était un foyer infectieux sur la troisième molaire mandibulaire gauche. L'atteinte médiastinale est ensuite le résultat d'une " coulée de cellulite " [2] le long des aponévroses cervicales à travers les trois principaux espaces anatomiques que sont l'espace rétroviscéral (ou rétropharyngé), la gouttière vasculaire et l'espace prétrachéal.

Comme c'est manifestement le cas ici, les germes responsables sont issus de la flore buccale endogène. En général, les anaérobies stricts sont majoritairement présents avec « 4 mousquetaires » que sont Prevotella, Porphyromonas gingivalis, Fusobacterium et les cocci anaérobies à Gram + (Dubreuil et Neut [5]). Ils sont très fréquemment associés à des streptocoques ${ }^{[5]}$. Les bacilles à Gram - sont rares. Le Staphylococcus aureus doit être pris en compte chez le toxicomane [5]. Le regroupement Spirochète-Fusobactérium constitue une association bien connue et redoutable ${ }^{[3]}$. Le traitement des cellulites aiguës diffuses repose en général sur l'association d'une antibiothérapie et d'un geste chirurgical. Devant la complexité de la bactériologie et de l'antibiothérapie, il est bien entendu souhaitable de recourir à un infectiologue. Le traitement doit être instauré de toute urgence. Compte tenu des délais de la culture bactériologique et de la complexité de l'identification des anaérobies, l'antibiothérapie est d'abord probabiliste dans un premier temps, et sera ensuite ajustée en fonction des résultats bactériologiques. L'antibiothérapie est administrée par voie veineuse et à dose élevée ; elle cible les streptocoques et les anaérobies issus de la flore endogène. L'amoxicilline est une molécule de choix dans le traitement des cellulites. Mais dans les infections graves, la bithérapie est souvent nécessaire pour les anaérobies, en dépit de la redondance des spectres. Elle permet d'élargir le spectre et de renforcer la bactéricidie ${ }^{[5]}$. On associe ainsi l'amoxicilline à l'acide clavulanique et/ou au métronidazole (Augmentin ${ }^{\circledast}$ seul ou

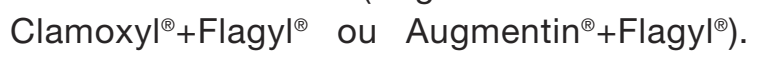
L'association amoxicilline-métronidazole est en général considérée comme un excellent choix dans le traitement des infections associant des streptocoques et des germes anaérobies [6]. En cas d'allergie aux bêta-lactamines, des macrolides, des streptogramines (pristinamycine) et des lincosamides (clindamycine) peuvent être prescrits ${ }^{[7]}$. On peut également associer de la clindamycine ou une fluoriquinolone à activité anaérobie comme la moxifloxacine, à un aminoglycoside ${ }^{[5]}$. L'AFSSAPS, dans son rapport de $2001{ }^{[8]}$, préconise la prescription de glycopeptides en cas d'allergie aux bêta-lactamines et/ou de résistance dans le cadre de ces infections sévères. Enfin, en cas de prélèvement positif pour les entérobacteries, on prescrit des céphalosporines injectables de troisième génération, très actives sur ces germes. Du fait de leur activité insuffisante sur les anaérobies, elles doivent toutefois être associées au métronidazole ${ }^{[5]}$. L'AFSSAPS ${ }^{[8]}$ évoque aussi la possibilité d'utiliser des céphalosporines en deuxième intention, après documentation microbiologique et antibiogramme.

Il faut bien sûr garder à l'esprit que l'antibiothérapie ne permet pas à elle seule d'obtenir la guérison, et doit nécessairement être associée à une intervention chirurgicale la plus précoce possible. Ce geste comporte le drainage, l'excision des tissus nécrosés et la mise à plat de toutes les zones cellulitiques. II nécessite une voie d'abord très large, de type cervicotomie bimastoïdienne (en forme de «U»), comme dans le cas rapporté. En médecine buccale chirurgie buccale VOL. $15, N^{\circ} 3$ 2009 page 123 


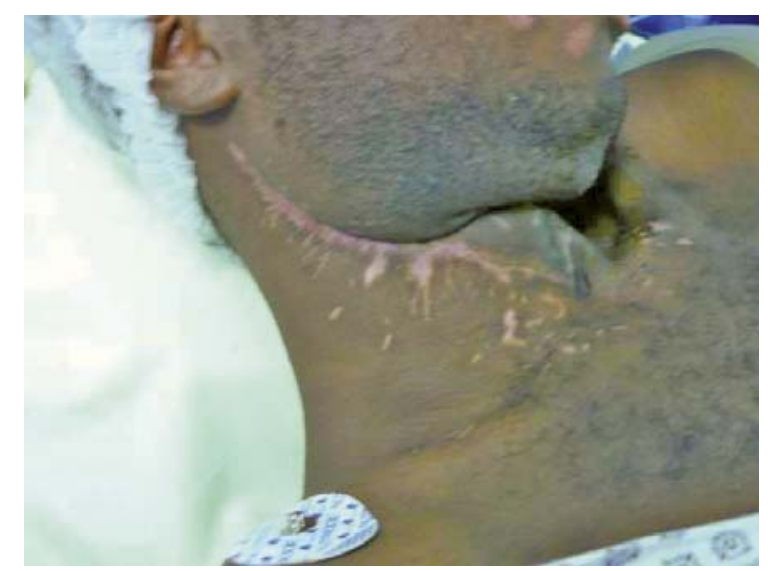

Figure 7 : Cicatrice cervicale à 2 mois. Cervical scar at 2 months.

cas d'extension médiastinale au dessous de la crosse aortique, un abord rétrosternal est indis- médecine buccale chirurgie buccale

VOL. $15, \mathrm{~N}^{\circ} 3$ 2009

page 124 pensable, ce qui a été nécessaire dans ce cas. Au sujet de la voie d'abord et du débridement, Romain et coll. ${ }^{[1]}$ estiment que l'on risque moins à en faire trop que pas assez. Après un bon lavage antiseptique, des lames de drainage sont mises en place. La plaie est laissée largement ouverte. II convient ensuite de pratiquer de grands lavages antiseptiques lors de chaque changement de pansement. Enfin, la fermeture cutanée intervient quand le processus infectieux est contrôlé. La régénération tissulaire laisse bien souvent des cicatrices dans les régions cervicale et thoracique, comme dans le cas rapporté (Fig. 7 et 8). Celles-ci peuvent être améliorées par la kinésithérapie (massage, vacuothérapie, douches filiformes, etc.).

Logiquement, l'extraction de la dent causale devrait intervenir le plus rapidement possible. Toutefois, dans le cas rapporté, l'équipe médicale a estimé que la priorité vitale revenait au drainage de l'infection cervico-médiastinale et non à l'avulsion de la dent causale. A ce stade, on peut effectivement considérer que la présence de la 38 ne jouait plus qu'un rôle mineur dans la progression de l'infection. La très large diffusion de cette infection et son ampleur témoignent du caractère autonome de son évolution. La dent causale n'a finalement été avulsée qu'après stabilisation de l'état de santé, à la fin du séjour dans le service de réanimation.

Les anti-inflammatoires non stéroïdiens (AINS) sont fréquemment désignés dans la littérature comme des facteurs susceptibles de favoriser la survenue ou la progression du processus infectieux. Dès 1989, Scheffer et coll. ${ }^{[4]}$ remarquent la mise en cause systématique des AINS dans la plupart des cas publiés. Muster [9] met en garde contre la prescription d'un AINS lors d'un processus septique. Chalom et Courrier ${ }^{[10]}$ estiment que dans le cas précis des cellulites d'origine dentaire, la prescription d'AINS est contre-indiquée car elle peut favoriser la survenue d'une flambée infectieuse. Rakotoarison et coll. [2] avancent l'hypothèse que l'augmentation d'incidence des cellulites graves serait en partie liée à l'automédication abusive avec des AINS. Mais il existe très peu d'études prospectives et comparatives sur le sujet. Celle de Bernard et coll. [11], portant sur un échantillon de 455 patients, ne retrouve pas d'augmentation de la mortalité dans le groupe traité par AINS. De sorte qu'on doit considérer que l'effet des AINS sur l'infection aigüe n'est pas établi. C'est précisément la conclusion qu'a tiré la Société Francophone de Médecine buccale et Chirurgie Buccale dans ses recommandations de 2008 [12]. De même, en 2001, la conférence de consensus de la Société Française de Dermatologie ${ }^{[13]}$ avait fait le constat que les données scientifiques ne permettaient pas d'établir de façon certaine que I'utilisation d'AINS au cours de dermo-hypodermites aiguës bactériennes pouvait favoriser la survenue des fasciites nécrosantes. A l'inverse, il n'est 
pas non plus démontré que la prise d'AINS n'a aucun effet sur l'évolution du processus infectieux, si bien que le débat n'est pas tranché. Et le cas rapporté ici, s'il illustre le problème, n'apporte bien entendu pas de solution. Par ailleurs il est bien établi que les AINS peuvent masquer les signes d'une infection. Le patient sous AINS sera donc amené à consulter plus tardivement, c'est-à-dire dans un état plus grave. Cette considération suffit peut-être à expliquer l'abondance des cas d'hospitalisation où le patient a consommé des AINS, d'autant que ces médicaments sont accessibles sans ordonnance. Dans le cas présent, la prescription d'ibuprofène qui avait été établie par le chirurgien-dentiste, comportait aussi un antibiotique. Compte tenu du contexte infectieux, même débutant, on peut estimer qu'une ordonnance d'AINS seul aurait été fautive.

Le patient du cas rapporté ici présente des séquelles fonctionnelles bucco-faciales. II mastique moins bien et l'auto-nettoyage des dents est donc moins efficace. La déglutition est également déficiente car la langue est déviée du fait de la lésion du nerf hypoglosse gauche; cette

\section{RÉFÉRENCES}

1 - Romain P, Schmidt P, Hannion X, Le Tarnec A, Chalumeau F, Legros M. Cellulites cervico-faciales gangréneuses d'origine dentaire : à propos de 11 cas. Rev Stomatol Chir Maxillofac $1989 ; 90$ : 428-37.

2 - Rakotoarison RA, Ramarozatovo NP, Rakoto FA, Rakotovao FJ-Cellulites cervico-faciales : à propos de 41 cas. Med Buccale Chir Buccale 2008 ; 14 : 35-9.

3 - Peron J.M., Mangez J.F.- Cellulites et fistules d'origine dentaire. Encycl Med Chir, Stomatol/Odontol 22-033A-10, Elsevier, Paris, 2002.

4 - Scheffer P, Ouazzani A, Esteban J, Lerondeau JC. Infections graves cervico-faciales d'origine dentaire. Rev Stomatol Chir Maxillofac 1989 ; 90 : 115-8.

5 - Dubreuil L, Neut C. Arguments microbiologiques pour optimiser l'antibiothérapie empirique des cellulites cervico-faciales. Med Buccale Chir Buccale 2005 ; 11 : 7 15.

6 - Timour Q. Antibiotiques : avantages et inconvénients des pénicillines seules versus association pénicillinesmétronidazole. Encycl Med Chir Stomatol 22-012-B11. Elsevier, Paris, 2007.

7 - Younes S, Roche Y. Face à l'infection bactérienne : quand, comment et quel antibiotique prescrire ? Réal Clin $2006 ; 17: 37-55$. déglutition altérée peut conduire à la stagnation d'aliments dans la cavité buccale. Par ailleurs, l'ouverture buccale limitée rend les manœuvres pour l'hygiène bucco-dentaire plus difficiles. On peut donc estimer que le risque carieux est augmenté. C'est cette considération qui a conduit à proposer une motivation pour l'hygiène buccodentaire et instituer des contrôles réguliers.

En conclusion, force est de constater que des infections à point de départ dentaire engageant le pronostic vital sont encore rencontrées en France, malgré un système de santé développé. Le coût humain est élevé : le patient conservera des séquelles esthétiques et fonctionnelles de cette infection. D'un point de vue social, la prise en charge a été lourde et coûteuse.

Le cas rapporté illustre la cascade de réactions biologiques conduisant à la défaillance de nombreuses fonctions de l'organisme. De nombreuses disciplines médicales ont été concernées : de l'urgence à la réanimation, de la cardiologie à la pneumologie, de la néphrologie à la dermatologie, de la chirurgie cervico-faciale et thoracique à l'odontologie.

8 -Agence Française de Sécurité Sanitaire et d'Accréditation des Produits de Santé- Prescription des antibiotiques en odontologie et stomatologie, 2001.

9 - Muster D. Médicaments de l'inflammation. Encycl Med Chir Stomatol 22-012-C-10. Elsevier, Paris, 2005.

10 - Chalom A, Courrier B. Face à l'inflammation : la prescription d'anti-inflammatoires est-elle nécessaire ? Réal Clin $2006 ; 17: 25-36$.

11 - Bernard GR, Wheeler AP, Russell JA, Schein R, Summer WR, Steinberg KP, Fulkerson WJ, Wright PE, Christman BW, Dupont WD, Higgins SB, Swindell BB. The effects of ibuprofen on the physiology and survival of patients with sepsis. The Ibuprofen in Sepsis Study Group. N Engl J Med 1997 ; 336 : 912-8.

12 - Société Francophone de Médecine Buccale et Chirurgie Buccale et Société Française de Cardiologie. Recommandations pour la prescription des anti-inflammatoires en chirurgie buccale chez l'adulte. Med Buccale Chir Buccale 2008 ; 14 : 129-59.

13 - Société Française de Dermatologie. Conférence de consensus : Érysipèle et fasciite nécrosante : prise en charge, 2001. médecine buccale chirurgie buccale VOL. $15, \mathrm{~N}^{\circ} 3$ 2009 page 125 